\title{
Cross-Ontological Analytics: Combining Associative and Hierarchical Relations in the Gene Ontologies to Assess Gene Product Similarity
}

\author{
C. Posse, A. Sanfilippo, B. Gopalan, R. Riensche, \\ N. Beagley, and B. Baddeley \\ Pacific Northwest National Laboratory \\ 902 Battelle Blvd, Richland, PO Box 999, WA 99352, USA \\ \{Christian.Posse, Antonio.Sanfilippo, Banu.Gopalan, \\ Rick.Riensche, Nathaniel.Beagley, Bob.Baddeley\} @pnl.gov
}

\begin{abstract}
Gene and gene product similarity is a fundamental diagnostic measure in analyzing biological data and constructing predictive models for functional genomics. With the rising influence of the gene ontologies, two complementary approaches have emerged where the similarity between two genes/gene products is obtained by comparing gene ontology (GO) annotations associated with the gene/gene products. One approach captures GO-based similarity in terms of hierarchical relations within each gene ontology. The other approach identifies GO-based similarity in terms of associative relations across the three gene ontologies. We propose a novel methodology where the two approaches can be merged with ensuing benefits in coverage and accuracy.
\end{abstract}

\section{Introduction}

The analysis of microarray data has become a mainstay of biological knowledge discovery, with a consequent explosive growth in the amount of DNA, RNA, and protein sequences and expression values to be interpreted. Gene and gene product similarity is a primary method that biologists use to carry out this interpretation.

When genes and gene products are represented by their sequences, gene and gene product similarity is usually performed using word-based heuristics to select maximal segment pair alignments similar to those implemented in BLAST [1] and FASTA [12] to approximate the Smith-Waterman algorithm [17]. Once gene or protein sequences are mapped into higher level representations, other similarity methods become available that provide additional insights to sequence similarity measures. For example, [13] demonstrate how gene product similarity can be performed in terms of PFAM domains (http://www.sanger.ac.uk/Software/Pfam) and [14] show how a computational analysis of scientific literature can be leveraged to define and recognize gene expression clusters.

With the rising influence of the gene ontologies (http://www.geneontology.org), new approaches have emerged where the similarity between genes or gene products is obtained by comparing gene ontology (GO) code annotations associated with them. The gene ontologies provide three orthogonal networks of functional genomic concepts structured in terms of semantic relationships such as inheritance and 
meronymy, which encode biological process (BP), molecular function (MF) and cellular component (CC) properties of gene and gene products. The use of GO code annotations in establishing gene and gene product similarity provides significant added functionality to methods such as BLAST and FASTA as it explicitly relates genes and gene products in terms of participation in the same/similar biological processes, presence in the same/similar cellular components and expression of the same/similar molecular functions.

Two main GO-based similarity approaches have emerged so far. One approach assesses GO code similarity in terms of shared hierarchical relations within each gene ontology (BP, MF, or CC) [2], [5], [9], [10]. For example, the relative semantic closeness of two biological processes would be determined by the informational specificity of the most immediate parent that the two biological processes share in the BP ontology. The other approach establishes GO code similarity by leveraging associative relations across the three gene ontologies [3]. Such associative relations make predictions such as which cellular component is most likely to be the location of a given biological process and which molecular function is most likely to be involved in a given biological process.

While these two approaches are fully complementary, no effort has been made so far to combine them. One major difficulty in carrying out such a combination resides in the heterogeneity of the two measures, one based on a hierarchical assessment and the other on an associative one. The aim of this paper is to present a methodology (XOA for Cross-Ontological Analytics) where this difficulty can be solved so that the two approaches can be integrated, with ensuing benefits in coverage and accuracy.

\section{Background}

GO-based similarity methods that focus on measuring intra-ontological relations have adopted the information theoretic treatment of semantic similarity developed in Natural Language Processing-see [4] for an extensive survey. An example of such an approach is given by [15], who defines semantic similarity between two concept nodes $c 1 \quad c 2$ in a semantic graph as the information content of the least common superordinate $(l c s)$ of $c l$ and $c 2$, as shown in (1) where $p(c)$ is the probability of encountering instances of $c$ in a specific corpus and $-\log p(c)$ indicates the information content of $c$.

$$
\operatorname{sim}(c 1, c 2)=-\log p(\operatorname{lcs}(c 1, c 2))
$$

[6] provide a refinement of Resnik's measure by factoring in the distance from each concept to the least common superordinate, as shown in (2).

$$
\operatorname{sim}(c 1, c 2)=-[2 * \log p(\operatorname{lcs}(c 1, c 2))-(\log p(c 1)+\log p(c 2))]
$$

[8] introduces a slight modification to Jiang's and Conrath's measure:

$$
\operatorname{sim}(c 1, c 2)=2 * \log p(\operatorname{lcs}(c 1, c 2)) /(\log p(c 1)+\log p(c 2))
$$

The information theoretic approach is very well suited to assess GO code similarity since each gene ontology is formalized as a directed acyclic graph, In addition, the GO database includes numerous curated GO annotations which can be used to 
calculate the information content of each GO code with high reliability. Evaluations of this methodology have yielded promising results. [9] and [10] demonstrate that there is strong correlation between GO-based similarity judgments for human proteins and similarity judgments obtained through BLAST searches for the same proteins. [2] show that there is a strong connection between the degree of GO-based similarity and the expression correlation of gene products.

As [3] remark, the main problem with the information theoretic approach to GO code similarity is that it does not take into account associative relations across the gene ontologies. For example, the two GO codes 0050909 (sensory perception of taste) and 0008527 (taste receptor activity) belong to different gene ontologies (BP and MF) but are undeniably very closely related. The information theoretic approach would simply miss associations of this kind as it is not designed to capture interontological relations.

[3] propose to recover associative relations across the gene ontologies using a variety of statistical techniques which estimate the similarity of two GO codes intraontologically in terms of the distribution of the gene product annotations associated with the two GO codes in the GO database. One such technique is an adaptation of the vector space model frequently used in Information Retrieval [16], where each GO code is represented as a vector of gene-based features weighted according to their distribution in the GO annotation database, and the similarity between two GO codes is computed as the cosine of the vectors for the two codes.

The ability to measure associative relations across the gene ontologies can significantly augment the functionality of the information theoretic approach so as to provide a more comprehensive assessment of gene and gene product similarity. However, in spite of their complementarity, the two GO code similarity measures are not easily integrated. This is because the two measures are obtained through different methods, express distinct senses of similarity (i.e. intra- and inter-ontological) and are thus incomparable. To date, this remains a challenge which is yet to be addressed.

\section{Cross-Ontological Analytics (XOA)}

Our basic approach in developing a GO-based similarity algorithm capable of combining intra- and inter-ontological relations consists in "translating" each associative relation across the gene ontologies into a hierarchical relation within a single ontology. More precisely, let $c 1(c 2)$ denote a GO code in the gene ontology $\mathrm{Ol}(\mathrm{O} 2)$, the XOA similarity between $c 1$ and $c 2$ is defined as follows:

$$
X O A(c 1, c 2)=\max \{\operatorname{sim}(c 1, c 3) * \cos (c 2, c 3), \operatorname{sim}(c 2, c 4) * \cos (c 1, c 4)\}
$$

where $\cos (c i, c j)$ denotes the cosine associative measure proposed by [3], $\operatorname{sim}(c i, c j)$ denotes any of the three intra-ontological semantic similarities defined in the previous section, see (1)-(3), and the maximum is taken over all GO codes $c 3$ in $O 1$ and $c 4$ in $O 2$. Note that if $O 1$ and $O 2$ refer to the same ontology, then:

$$
X O A(c 1, c 2)=\operatorname{sim}(c 1, c 2) .
$$

This shows that the XOA approach extends intra-ontological similarities to the interontological context. Conversely, $c 3(c 4)$ can be seen as a surrogate for $c 2(c 1)$ in the 
same ontology as $c l(c 2)$. The inter-ontological semantic similarity between $c 1(c 2)$ and $c 2(c 1)$ can be then estimated from the intra-ontological semantic similarity between $c 1(c 2)$ and $c 3(c 4)$ and the associative relation between $c 3(c 4)$ and $c 2(c 1)$.

The XOA similarity between genes/gene products derives from the XOA similarity between GO codes. Let $G P 1$ and $G P 2$ be two genes/gene products. Let $c 11, c 12, \ldots$, $c 1 n$ denote the set of GO codes associated with $G P 1$ and $c 21, c 22, \ldots, c 2 m$ the set of GO codes associated with $G P 2$. The XOA similarity between $G P 1$ and $G P 2$ is given as shown in (6), where $i=1, \ldots, n$ and $j=1, \ldots, m$.

$$
X O A(G P 1, G P 2)=\max \{X O A(c 1 i, c 2 j)\}
$$

Note that [10] use the average instead of the maximum. However, genes/gene products with many GO annotations have higher chance of exhibiting a few lower XOA similarities between GO codes. This automatically lowers the similarity between genes/gene products. In our experiments, using the maximum provided more consistent results than the average. [2] also find that the average does not always meaningfully estimate similarity and plan on investigating the maximum approach. The maximum approach is also consistent with the first information content based semantic similarity proposed by [15] where the similarity between two words is defined as the maximum of the similarities between all concept pairs emerging from difference senses for the two words.

\section{Evaluation}

To evaluate the XOA approach, we follow the benchmarking study carried out by [10], which compares intra-ontological semantic similarities with sequence similarities as measured with BLAST. In doing so, we use current data from the GO database. This has several advantages. First, the replication of the above benchmarking study three years later lets us assess the effects of the changes of the gene ontologies and reference databases on the findings unearthed by [10]. Second, it allows us to improve the benchmarking analysis. Finally, it allows us to compare our XOA results with the intra-ontological semantic similarities obtained by [10].

All results reported are from analyses carried out on the May 2005 release of GO database available from http://www.godatabase.org/dev. A large set of protein pairs with varying degrees of sequence similarities was obtained by

1) performing BLAST searches with expect value of 100 over the set of approximately 7000 SWISS-PROT human proteins for which GO codes were available, and

2) retaining proteins from BLAST results for which GO codes were available. Only those codes with "Traceable Author Statement" tags were used.

The full set of BLAST searches was completed in less than 30 minutes using ScalaBLAST [11], a powerful parallel BLAST engine. This process generated a set of 255,529 protein pairs. Table 1 summarizes the distribution of GO codes in the benchmarking set. It reveals that XOA provides coverage for around 15\% more protein pairs than the combination of intra-ontological semantic similarities applied separately to the $\mathrm{BP}, \mathrm{MF}$ and $\mathrm{CC}$ ontologies. When compared to any single gene ontology the XOA coverage is at least twice as large. 
Table 1. Distribution of TAS GO codes in benchmarking set

\begin{tabular}{|l|r|}
\hline \multicolumn{1}{|c|}{ GO Code Distribution } & Number of protein pairs \\
\hline Each protein in the pair has at least one GO code & 255,529 \\
\hline Each protein in the pair has at least one BP GO code & 128,921 \\
\hline Each protein in the pair has at least one MF GO code & 112,027 \\
\hline Each protein in the pair has at least one CC GO code & 94,677 \\
\hline $\begin{array}{l}\text { Both proteins in the pair have at least one GO code } \\
\text { in the same ontology (BP, MF or CC) }\end{array}$ & 216,714 \\
\hline $\begin{array}{l}\text { Both proteins in the pair have only GO codes in } \\
\text { different ontologies }\end{array}$ & 38,788 \\
\hline
\end{tabular}

[9] and [10] compare semantic and sequential similarities by calculating the correlation coefficient between the intra-ontological semantic similarities and the logarithm of the BLAST bit score LBS for each of the gene ontologies. Table 2 provides these correlations based on our more recent data. In addition, it shows the correlation between XOA and LBS.

Table 2. Correlation coefficients between LBS and semantic similarities. Correlation coefficients were calculated from the subset of proteins given in Table 1.

\begin{tabular}{|l|l|l|c|}
\hline Gene Ontology & Resnik & Lin & Jiang and Conrath \\
\hline Molecular Function & 0.555 & 0.516 & 0.468 \\
\hline Biological Process & 0.394 & 0.394 & 0.378 \\
\hline Cellular Component & 0.318 & 0.345 & 0.320 \\
\hline XOA & 0.412 & 0.362 & 0.270 \\
\hline
\end{tabular}

We observe that despite the major changes in the gene ontologies in the last 3 years and the enrichment of the SWISS-PROT database these correlations are very similar to those produced by [10] in 2003 for the intra-ontological semantic similarities. This is confirmed in Figure 1, which shows the functional relationships between LBS and the semantic similarities. Furthermore, Table 2 agrees with [10] findings that Resnikbased semantic similarities on one hand and the Molecular Function ontology on the other end tend to produce higher correlations. A novel finding is that XOA correlations are comparable to those obtained with intra-ontological semantic similarities though they are based on at least $100 \%$ more protein pairs than any single ontology comparison.

These results however should be taken with some caution. Correlation coefficients can only measure linear relationship between two variables. If taking the logarithm of the bit score straightens the relationship between the Resnik similarity measure and the bit score, this is not the case for the other similarity measures. One can show that, for the Lin and Jiang \& Conrath measures, higher order transformations of the bit score, such as $\mathrm{a}+\mathrm{b} * \mathrm{LBS}+\mathrm{c} * \mathrm{LBS}^{2}$ with $\mathrm{a}, \mathrm{b}$ and $\mathrm{c}$ being some constants, are required in order to get a linear relationship. Rather than pursuing this direction we note that what we are truly interested in measuring is a monotonic relationship between the bit score 

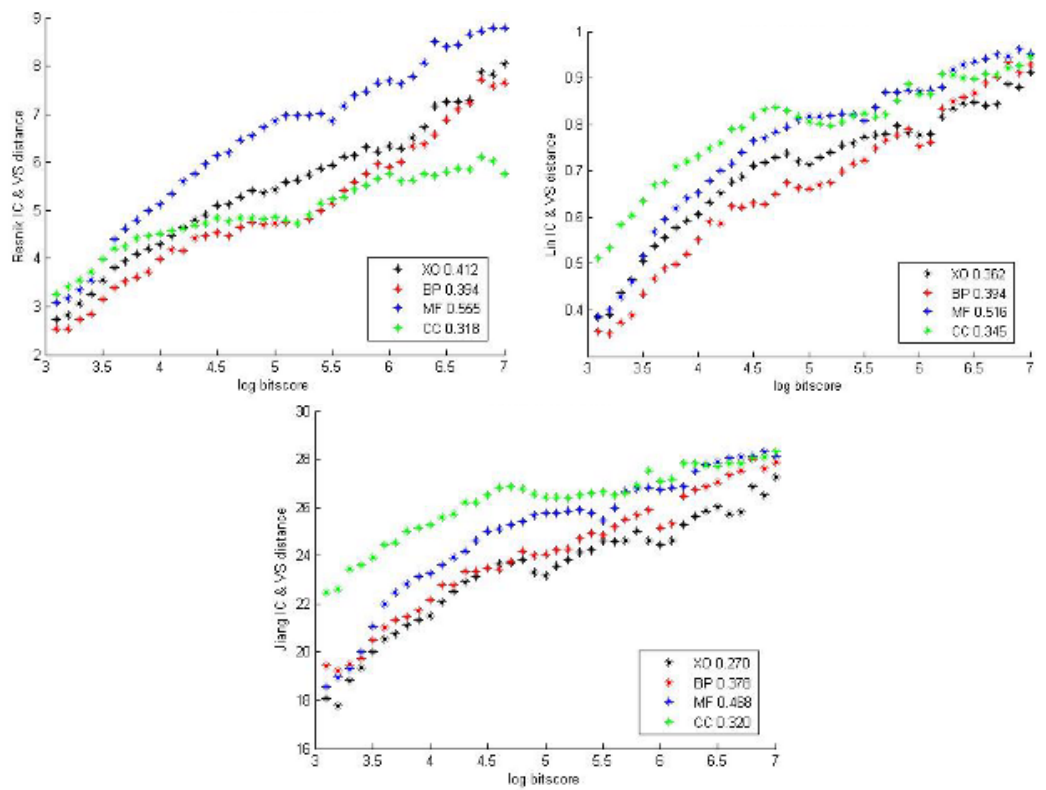

Fig. 1. Comparing sequence and semantic similarity. BLAST search were performed for each SWISS-PROT human protein, and matches were analyzed for semantic similarity with the search protein. Following [10], intervals were taken along the $\mathrm{x}$-axis and $\log [\mathrm{bit}$ score] and semantic similarity values were averaged.

Table 3. Spearman rank order correlation coefficients between bit score and semantic similarities. Correlation coefficients were calculated from the subset of proteins given in Table 1 .

\begin{tabular}{|l|l|l|c|}
\hline Gene Ontology & Resnik & Lin & Jiang and Conrath \\
\hline Molecular Function & 0.575 & 0.544 & 0.516 \\
\hline Biological Process & 0.389 & 0.417 & 0.423 \\
\hline Cellular Component & 0.343 & 0.387 & 0.382 \\
\hline XOA & 0.405 & 0.393 & 0.368 \\
\hline
\end{tabular}

and the semantic similarity. A well-known measure of monotonicity is Spearman rank order correlation coefficient [7]. Table 3 reports this coefficient for the configuration set in Table 2.

One can see that correlations tend to increase using the Spearman rank order correlation coefficient. Even more interesting is the fact that each similarity measure yields best correlation results when applied to a different ontology: Resnik for MF, Jiang and Conrath for BP and Lin for CC. This is not too surprising. Because Resnik measure rests only on the closest parent node of the node pair, it will tend to produce higher values in well developed ontologies, such as MF, where nodes tend to be more specific. By normalizing the Resnik measure with information content of the node pair, the Lin and Jiang \& Conrath measures prefer sparser ontologies with fewer child nodes. Because Jiang and Conrath measure normalizes via a difference and Lin 
measure via a ratio, the former favors more uniform ontologies while the latter prefers ontologies more developed in regions of higher specificity within the ontology.

Another issue with the results in Tables 2 and 3 is that they do not reflect the significant difference in coverage across the different methods. XOA correlations rely on all protein pairs for which there are GO codes available. By contrast, MF- and BPbased correlations use only half of them and CC-based correlations use only a third. Though the three gene ontologies are orthogonal, they can be subsumed under a single root level term whose information content is 0. Conceptually, this root node allows us to compute the Resnik, Lin and Jiang \& Conrath semantic similarities for nodes in different gene ontologies. In practice, this always results in a zero value for the Resnik and Lin similarities and very small values for the Jiang and Conrath similarity. Using this mechanism, we computed Spearman rank order correlations between the bit score and the various semantic similarities using all protein pairs in our benchmarking set. Table 4 summarizes these values. All correlations decrease except for the XOA ones which remain unchanged and become the strongest ones.

Table 4. Spearman rank order correlation coefficients between bit score and semantic similarities. Correlation coefficients were calculated using the entire benchmarking set of 255,529 protein pairs.

\begin{tabular}{|l|l|l|c|}
\hline Gene Ontology & Resnik & Lin & Jiang and Conrath \\
\hline Molecular Function & 0.307 & 0.301 & 0.296 \\
\hline Biological Process & 0.195 & 0.202 & 0.203 \\
\hline Cellular Component & 0.229 & 0.234 & 0.233 \\
\hline XOA & 0.405 & 0.393 & 0.368 \\
\hline
\end{tabular}

\section{Conclusions and Future Work}

Our XOA approach provides a more comprehensive approach to the use of GO annotations for the assessment of gene and product similarity than any of the methods previously proposed. Due to its ability to yield a greater number of GO code comparisons, XOA accounted for $15 \%$ more human protein pairs than the combined three intra-ontological semantic similarity approaches (see Table 1), with a consequent improvement of the overall correlation with BLAST results (see Table 4).

Moving forward, we are planning the use of textual evidence extracted from relevant biomedical literature to improve inter-ontological GO-based similarities. We also intend to use manually curated ground truth datasets such as the PFAM database (http://www.sanger.ac.uk/Software/Pfam/) in addition to BLAST gene (product) similarity search results, to carry out a more meaningful evaluation of XOA. Finally, we have started to engage XOA in the analysis of gene expression data to aid the identification of biological pathways and the detection of biomarkers. These applications are yielding promising results and will constitute the ultimate testing ground for XOA. 


\section{References}

1. Altschul, S.F., T. L. Madden, A. A. Schaffer, J. Zhang, Z. Anang, W. Miller and D.J. Lipman (1997) Gapped BLAST and PSI-BLST: a new generation of protein database search programs. Nucl. Acids Res. 25:3389-3402.

2. Azuaje F., H. Wang and O. Bodenreider (2005) Ontology-driven similarity approaches to supporting gene functional assessment. In Proceedings of the ISMB'2005 SIG meeting on Bio-ontologies 2005, pages 9-10.

3. Bodenreider, O., M. Aubry and A. Burgun (2005) Non-lexical approaches to identifying associative relations in the gene ontology. In Proceedings of Pacific Symposium on Biocomputing, pages 104-115.

4. Budanitsky, A. (1999) Lexical semantic relatedness and its application in natural language processing. Technical report CSRG-390, Department of Computer Science, University of Toronto.

5. Couto, F. M., M. J. Silva and P. Coutinho (2003) Implementation of a functional semantic similarity measure between gene-products. Technical Report, Department of Informatics, University of Lisbon, http://www.di.fc.ul.pt/tech-reports/03-29.pdf.

6. Jiang J. and D. Conrath (1997) Semantic similarity based on corpus statistics and lexical taxonomy. In Proceedings of International Conference on Research in Computational Linguistics, Taiwan.

7. Kendall M. G. (1949) Rank and product-moment correlation. Biometrika 36: 177-193.

8. Lin, D. (1998) An information-theoretic definition of similarity. In Proceedings of the 15th International Conference on Machine Learning, Madison, WI.

9. Lord P.W., R.D. Stevens, A. Brass, and C.A.Goble (2002) Investigating semantic similarity measures across the Gene Ontology: the relationship between sequence and annotation. Bioinformatics 19(10):1275-1283.

10. Lord P.W., R.D. Stevens, A. Brass, and C.A.Goble (2003) Semantic similarity measures as tools for exploring the Gene Ontology. In Proceedings of Pacific Symposium on Biocomputing, pages 601-612.

11. Oehmen C.S. and J.N. Nieplocha (2006) ScalaBLAST: A scalable implementation of BLAST for high performance data-intensive bioinformatics analysis. IEEE Transactions on Parallel and Distributed Systems, in press.

12. Pearson, W. R. and D. J. Lipman (1988) Improved tools for biological sequence analysis. In Proceedings of the National Academy of Sciences 85:2444-2448.

13. Popescu, M., J. M. Keller and J.A. Mitchell (2005) Gene ontology automatic annotation using a domain based gene product similarity measure. In Proceedings of 14th IEEE International Conference on Fuzzy Systems, Reno, Nevada, May 21-25, pages 108-111.

14. Raychaudhuri, S., J. T. Chang, F. Imam and R. B. Altman (2003) The computational analysis of scientific literature to define and recognize gene expression clusters. Nucleic Acids Research 31(15):4553-4560.

15. Resnik, P. (1995) Using information content to evaluate semantic similarity. In Proceedings of the 14th International Joint Conference on Artificial Intelligence, pages 448-453, Montreal.

16. Salton, G., A. Wong and C. S. Yang (1975) A Vector space model for automatic indexing, CACM 18(11):613-620.

17. Smith, T. and M. S. Waterman (1981) Identification of common molecular subsequences. J. Mol. Biol. 147:195-197. 